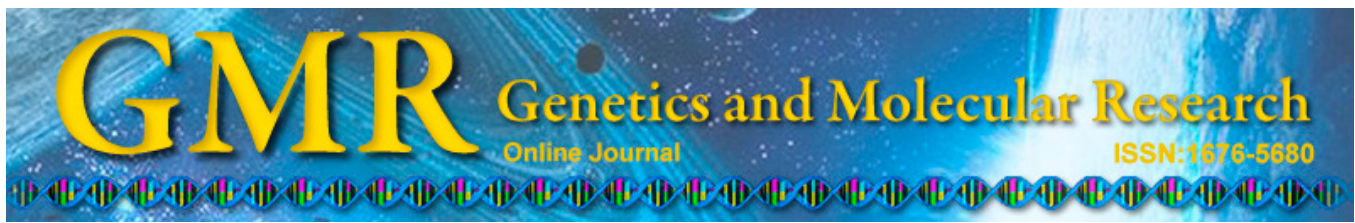

\title{
Human chorionic gonadotropin promotes expression of protein absorption factors in the intestine of goldfish (Carassius auratus)
}

\author{
Y. Zhou ${ }^{1,2 *}$, G. Hao ${ }^{1 *}$, H. Zhong ${ }^{2}$, Q. Wu ${ }^{1}$, S.Q. Lu ${ }^{1}$, Q. Zhao ${ }^{1}$ and Z. Liu ${ }^{1}$ \\ ${ }^{1}$ Department of Biotechnology and Environmental Science, \\ Changsha University, Changsha, Hunan, China \\ ${ }^{2}$ Guangxi Key Laboratory of Aquatic Genetic Breeding and Healthy Aquaculture, \\ Guangxi Academy of Fisheries Science, Nanning, Guangxi, China \\ *These authors contributed equally to this study. \\ Corresponding author: Z. Liu \\ E-mail:25300085@qq.com
}

Genet. Mol. Res. 14 (3): 8306-8313 (2015)

Received October 13, 2014

Accepted April 27, 2015

Published July 27, 2015

DOI http://dx.doi.org/10.4238/2015.July.27.19

\begin{abstract}
Protein use is crucial for the ovulation and spawning of fish. Currently, limited information is available regarding the expression of protein absorption factors during the breeding seasons of teleosts and thus how various proteins involved in this process is not well-understood. The expression of CDX2, CREB, gluatamate dehydrogenase, LAT2, aminopeptidase N, PepT1, and SP1 were significantly elevated from the non-breeding season to the breeding season in female goldfish, and all proteins except PepT1 and SP1 were elevated in male goldfish. Injection of human chorionic gonadotropin upregulated the expression of all proteins except for aminopeptidase $\mathrm{N}$ in female goldfish and SP1 in male goldfish, suggesting a luteinizing hormone-inductive effect on protein absorption factors. Protein use in the intestine is increased during the breeding seasons as a result of increased luteinizing hormone.
\end{abstract}

Key words: Goldfish; Human chorionic gonadotropin; Intestine; Hypothalamic-pituitary-gonad axis 


\section{INTRODUCTION}

Unlike other vertebrate taxa, fish require high protein concentrations in their diets to obtain the high levels of amino acids required for ammonotely and poikilothermy associated with their aquatic mode of life (Sire and Vernier, 1992). There are 2 main steps in the metabolism and use of amino acids in the intestine of teleosts. First, amino acids produced by enzymolysis of proteins are deaminated and transaminated into other metabolic intermediates. Second, the intermediates are used for protein synthesis. Aminopeptidase N (APN) (Sherriff et al., 1992) and glutamate dehydrogenase (GDH) (Liu et al., 2012) play crucial roles in the digestion of peptides. APN catalyzes N-terminal amino acid enzymolysis, which transforms small peptides into amino acids. GDH catalyzes the reversible deamination of glutamate, producing ammonia as a byproduct, and participates in the nitrogen cycle. A number of studies have indicated that protein digestion products such as small peptides and amino acids are transferred to the cell interior through specific transporters. PepT1 has been found to be a transporter of di- and tri-peptides (Brandsch et al., 2004). Furthermore, PepT1 regulation is mediated by the SP1 and CDX2 transcription factors (Dalmasso et al., 2008). These factors contribute to basal, intestine-specific, and fasting-induced PepT1 expression (Dalmasso et al., 2008). LAT2 is a specialized transporter that facilitates the transfer of amino acids across epithelial cell layers (Fotiadis et al., 2013).

In teleosts, as in other vertebrates, reproductive activities are regulated by the hypothalamic-pituitary-gonad (HPG) axis (Maruska and Fernald, 2011). In artificial pools, female fish often exhibit reproductive dysfunction because of inadequate luteinizing hormone (LH) secretion by the pituitary (Zohar and Mylonas, 2001). Thus, human chorionic gonadotropin (hCG), an analog of $\mathrm{LH}$, is sometimes employed to manipulate ovulation (Mylonas et al., 2010). Additionally, stimulation of fish by hCG injection was found to be a valuable approach for studying gene activation during the spawning period.

Here, we report the effect of hCG on protein absorption factors in the intestine of goldfish (Carassius auratus). To investigate changes in protein absorption during gonadal development, we determined the mRNA expression of PepT1, CDX2, CREB, GDH, LAT2, $\mathrm{APN}$, and SP1 during this process using real-time polymerase chain reaction (PCR). Further, to understand the mechanisms of the changes during this process, injection of hCG was performed to examine its effect on these protein absorption factors in the intestine of goldfish.

\section{MATERIAL AND METHODS}

\section{Fish samples}

Goldfish were provided by the Hunan Institute of Aquatic Science. After the fish were anesthetized with 2-phenoxyethanol (Sigma-Aldrich, St. Louis, MO, USA), intestinal tissues were excised and immediately frozen in liquid nitrogen and stored at $-80^{\circ} \mathrm{C}$ until further analysis.

\section{Real-time PCR}

Total RNAs of intestinal tissues were isolated using TRIzol reagent (Invitrogen, Carlsbad, CA, USA). Subsequently, first-strand cDNAs were synthesized using AMV reverse transcriptase (Fermentas, Vilnius, Lithuania). The primers were designed as shown in Table 1. $\beta$-actin was used as an endogenous control. The PCR conditions were: $50^{\circ} \mathrm{C}$ for $5 \mathrm{~min}$ and 
$95^{\circ} \mathrm{C}$ for $10 \mathrm{~min}$, followed by 40 cycles at $95^{\circ} \mathrm{C}$ for $15 \mathrm{~s}$, and $60^{\circ} \mathrm{C}$ for $45 \mathrm{~s}$. After the amplifications, dissociation curves were constructed to confirm that the products were specific. Relative gene expression was calculated by the $2^{-\Delta \Delta C t}$ method using the SDS software v1.3.1 (Applied Biosystems, Foster City, CA, USA).

\begin{tabular}{ll}
\multicolumn{1}{c}{ Table 1. Primers used for quantitative RT-PCR. } & \\
\hline Primer name & Sequences (5' to $\left.3^{\prime}\right)$ \\
\hline APN-sense & GCCCAAAACTGTGCAAGAAAT \\
APN-antisense & CTCTGATGCGTGTCTGGTTTAGT \\
GDH-sense & CACAACACAGTCAGCACAGAACTC \\
GDH-antisense & ACCAAATGGCACATCCACAACAG \\
Pept1-sense & CAGGTTTGTGATGGCTTTAGG \\
Pept1-antisense & TGTGGATGTCGTTCGGGTTA \\
LAT2-sense & TCTGTTCCCCACCTGCTTCC \\
LAT2-antisense & CCCTGTAGAAATGCTAATGCG \\
CDX2-sense & GAACCCTCAGAATTTTGTACCCG \\
CDX2-antisense & GGTAATTCCAGGGACGTGATG \\
CREB-sense & TCAATCAGTCCCACGGATAGAA \\
CREB-antisense & GTCTGTAGCCCTTGAACTCCAT \\
SP1-sense & TGGCTGCGGGAAGGTT \\
SP1-antisense & CATCCAGGTGCAGACAAAGGG \\
$\beta$-actin & GCTCTTCCCCATGCAATCCT \\
$\beta$-actin & GGTTCCCATCTCCTGCTCAA \\
\hline
\end{tabular}

\section{Seasonal changes in absorption factors}

Goldfish in the non-breeding season (1.5 years old, November, 4 females and 4 males, mean body weight $320.1 \pm 44.5 \mathrm{~g}$ ) and in the breeding season ( 2 years old, March, 4 females and 4 males, mean body weight $383.2 \pm 54.7 \mathrm{~g}$ ) were collected. The intestine of each fish was removed and used for individual real-time PCR assays of PepT1, CDX2, CREB, GDH, LAT2, APN, and SP1.

\section{hCG effects on protein absorption factors}

Twenty female adult individuals and 20 male adult individuals were randomly divided into 5 treatment groups: female control group, $0.6 \mathrm{IU} / \mathrm{g}$ hCG at 1 day post-injection group, $0.6 \mathrm{IU} / \mathrm{g}$ hCG at 7 days post-injection group, $3 \mathrm{IU} / \mathrm{g}$ hCG at 1 day post-injection group, and 3 $\mathrm{IU} / \mathrm{g}$ hCG at 7 days post-injection group. The control groups and hCG treatment groups were injected with saline, 0.6 and $3 \mathrm{IU} / \mathrm{kg}$ body weight hCG, respectively. Each group was stored in vehicle at $\left(23^{\circ} \mathrm{C}\right)$ until the intestines were collected.

\section{Statistical analysis}

The data were analyzed by one-way analysis of variance. P values $<0.05$ were considered to indicate significant differences. All statistical analyses were performed using the SPSS 13.0 software (Chicago, IL, USA).

\section{RESULTS}

The expression of all protein absorption factors increased significantly in both female 
and male fish from the non-breeding season to the breeding season, except for PepT1 and SP1 in male fish (Figure 1). These observations suggest that the protein-absorbing capacity of the intestine was improved during the breeding season in both female and male fish.
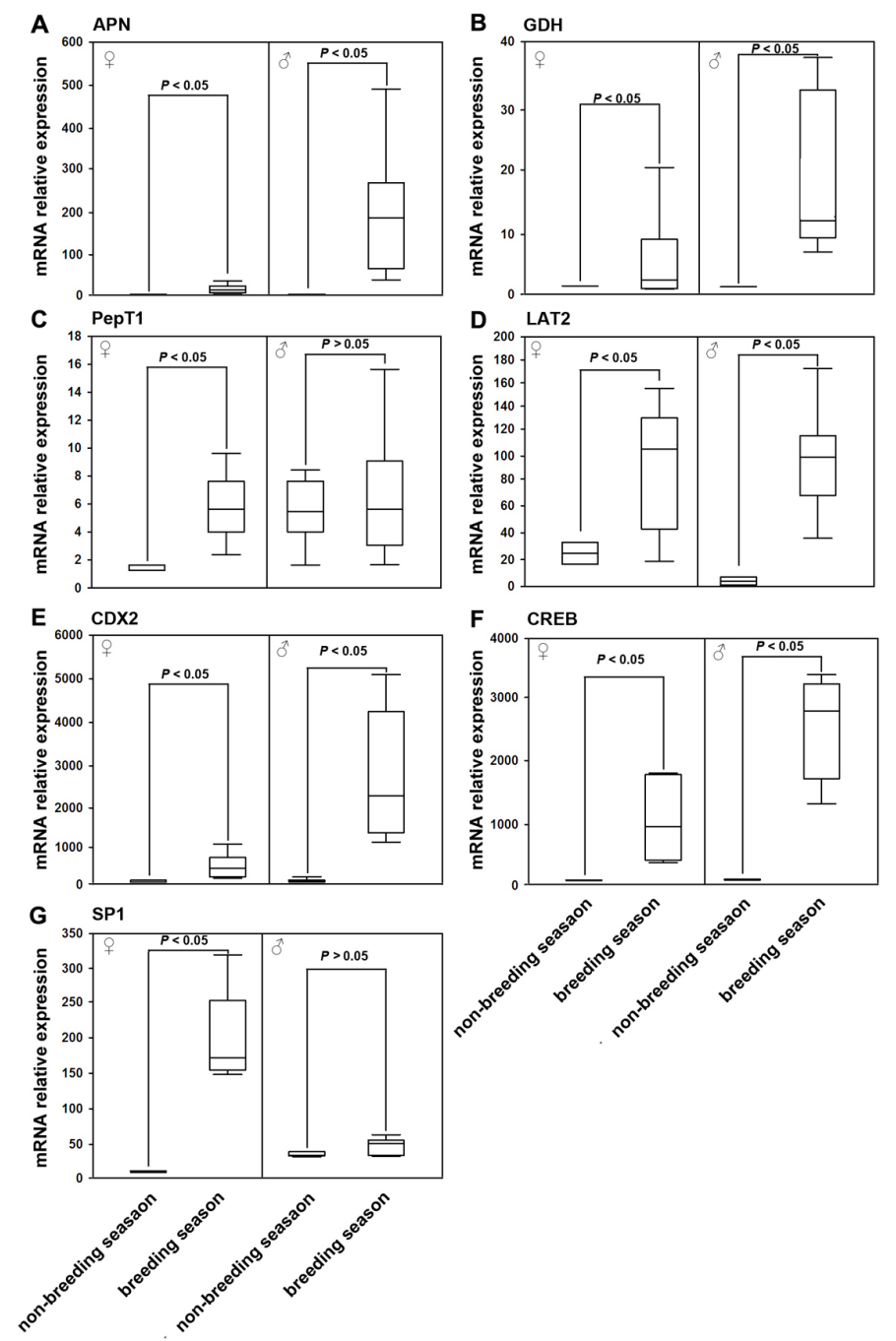

Figure 1. Comparison of the expression levels of APN, GDH, PepT1, LAT2, CDX2, CREB, and SP1 (A-G) between breeding and non-breeding seasons of female and male Carassius auratus. Relative expression was determined by real-time PCR and normalized using $\beta$-actin. $P$ values $<0.05$ indicate significant differences between breeding and non-breeding mean values (one-way analysis of variance). Data are reported as means $\pm \mathrm{SD}(\mathrm{N}=4)$.

After injection with hCG, no significant increase in APN expression in the intestine was observed among the female groups, but a significant increase in transcription was observed at 1 day post-injection in male fish (Figure 2A). In contrast, expression of GDH and PepT1 showed significant increases 1 and 7 days after hCG injection in both male and female fish (Figure 2B, 
C). The expression levels of LAT2 were significantly enhanced in female fish at 1 day post-injection of $0.6 \mathrm{IU} / \mathrm{g}$ hCG and at 1 and 7 days post-injection of $3 \mathrm{IU} / \mathrm{g}$ hCG. Increased expression of LAT2 was also observed in male fish after hCG injection (Figure 2D). The transcripts of CDX2 were significantly increased 7 days post-injection of $3 \mathrm{IU} / \mathrm{g} \mathrm{hCG}$ in intestines of both female and male fish (Figure 2E). The expression of CREB in the intestine was significantly upregulated only by $3 \mathrm{IU} / \mathrm{g}$ hCG at 1 day post-injection in female fish and at 7 days post-injection in male fish (Figure 2F). In female fish, SP1 also showed a significant increase at 1 and 7 days post-injection with $0.6 \mathrm{IU} / \mathrm{g}$ hCG and at 1 day post-injection with $3.0 \mathrm{IU} / \mathrm{g} \mathrm{hCG}$. However, no significant differences in SP1 were observed after either 0.6 or $3.0 \mathrm{IU} / \mathrm{g} \mathrm{hCG}$ injection in male fish (Figure 2G).
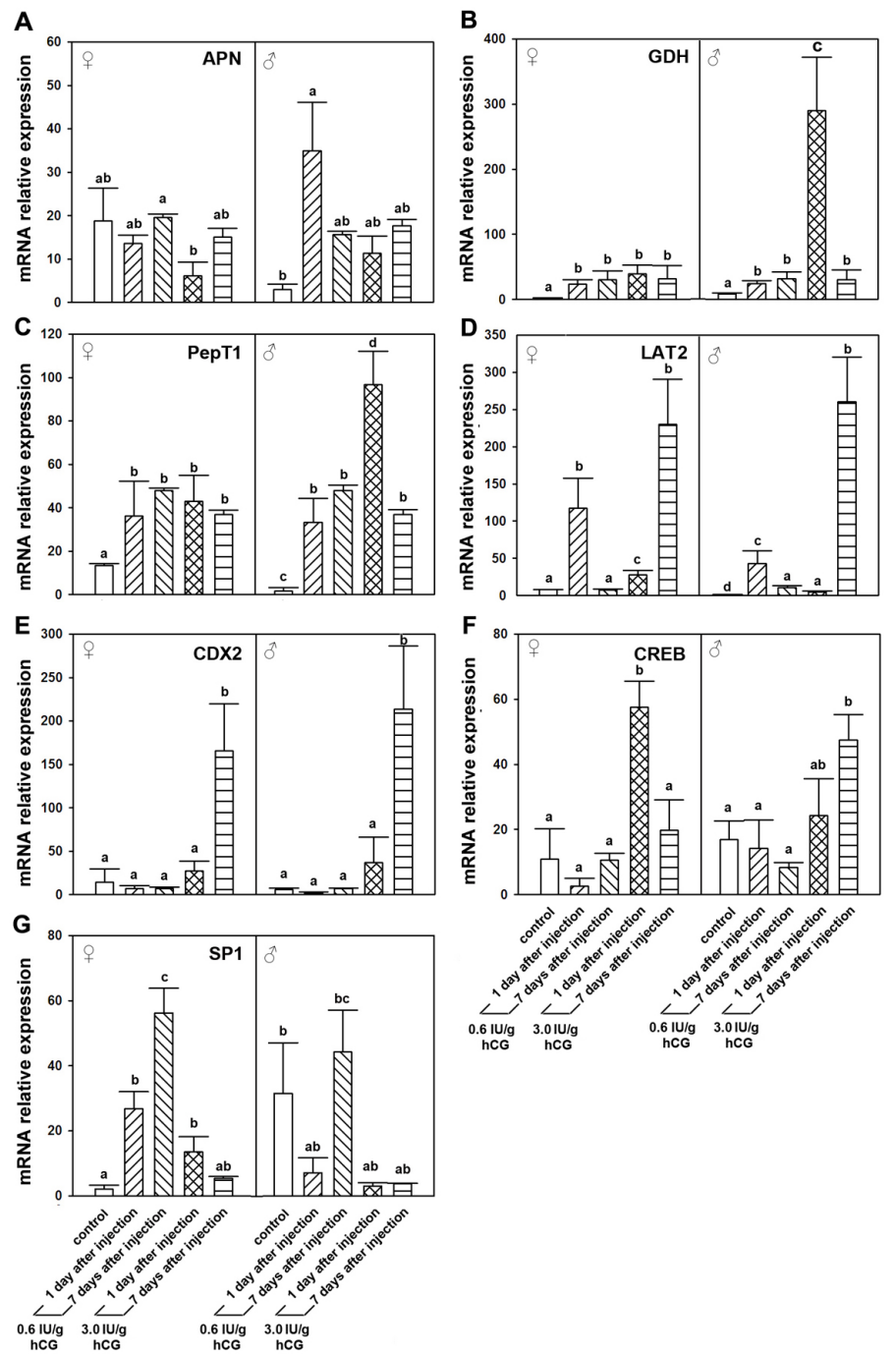

$0.610 / 9$
hCG $\underset{\text { hCG }}{3.01 U / 9}$

$\underset{\mathrm{hCG}}{0.6 \mathrm{IU/g}} \underset{\mathrm{hCG}}{3.01 \mathrm{U} / \mathrm{g}}$

Figure 2. Real-time PCR of the APN, GDH, PepT1, LAT2, CDX2, CREB, and SP1 genes after hCG injection (AG). The data are normalized relative to the expression of $\beta$-actin. Data are reported as means $\pm \mathrm{SD}(\mathrm{N}=4)$; bars labeled with different letters are significantly different (one-way analysis of variance, $\mathrm{P}<0.05$ ). 


\section{DISCUSSION}

Several studies have demonstrated changes in the protein-absorbing capacity of the intestine during development. In rats, the expression levels of PepT1 were high in juveniles at 3-5 days after birth (Shen et al., 2001). In chickens, LAT2 and PepT1 were upregulated between embryonic day 18 and 14 days after hatching (Li et al., 2008). However, little information is available regarding their expression during breeding stages. In this study, we demonstrated a significant increase in APN, GDH, SP1, and CDX2 in the intestine of goldfish, suggesting that these factors are induced in the intestine during development. To elucidate the basis of this upregulation of expression, we examined seasonal differentiation in protein absorption factors followed by analysis of the influence of the HPG axis on these factors by injecting hCG. In the present study, hCG promoted the activities of nearly all protein absorption factors in the intestine of goldfish with the exception of APN in female fish and SP1 in male fish. We also observed that the optimal injection dose and time for promotion of these protein absorption factors varied among different genes. This may be because of negative or positive feedback regulation within the HPG axis. Therefore, hCG induced the protein absorption in the intestine, suggesting that the HPG axis promotes protein absorption during the breeding season.

A high-protein concentration in the diet is a key requirement for achieving rapid growth rates in the fish culture industry. The function and regulation of protein absorption factors have been clearly demonstrated. However, during the breeding season, we observed that a high-protein diet is not suitable for obtaining high-quality eggs or sperm. A highprotein diet may contribute to a low gonad index and fat deposition. Intriguingly, the present study showed that protein absorptive capacity increased sharply during the breeding season. APN and GDH participate in the digestion of proteins into amino acids and small peptides (Liu et al., 2012). During the breeding season, fish require an abundant supply of amino acids for egg and sperm production. The main components of eggs and sperm are spermatogonial protein and vitellogenin. The augmented digestive capacity associated with increased APN and GDH levels may play a crucial role in this process. Similarly, PepT1 and LAT2, which are transporters of small peptides and amino acids, are key factors that increase the transporting capacity of the intestine during breeding season. As previously reported, in the intestine, most protein-absorption-related genes are regulated by transcription factors, such as CDX2, CREB, and SP1 (Dalmasso et al., 2008). Accordingly, these transcription factors were sharply upregulated in the intestine during the breeding season. Our results showing increased protein absorption in the intestine during the ovulation was corroborated by the results following hCG injection. Increased LH during the breeding season induces protein absorption factors in the intestine of goldfish, including APN, GDH, PepT1, SP1, CDX2, and LAT2. Consequently, the high protein-absorbing ability adapted because of the large amounts of amino acids required for egg and sperm formation, as well as for ovulation and spawning (Figure 3).

In summary, the increased expression of protein absorption factors induced by $\mathrm{LH}$ secretion during the breeding season promotes the protein-absorbing capacity of the intestine of goldfish. These observations provide a foundation for future breeding studies aimed at improving protein absorption. 


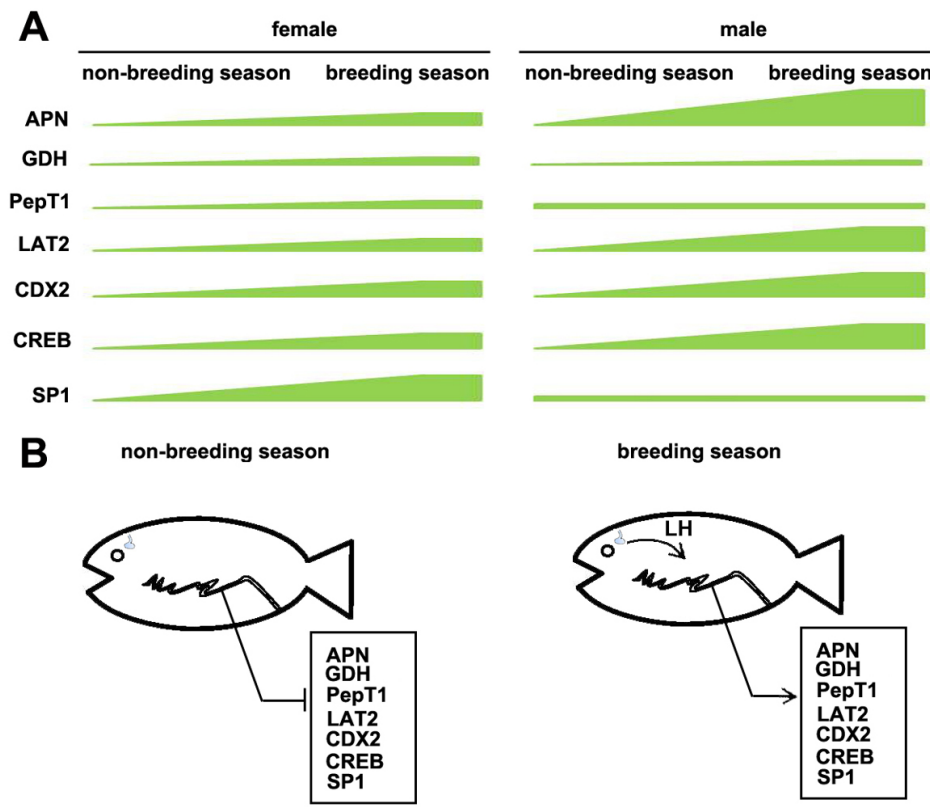

Figure 3. Seasonal changes (A) and LH regulation (B) of protein absorption factors in goldfish. A. Most factors sharply increase during breeding season except for PepT1 and SP1 in male fish. B. During the breeding season, the pituitary secretes $\mathrm{LH}$, which promotes protein absorption factors in the intestine.

\section{ACKNOWLEDGMENTS}

Research supported by the National Natural Science Foundation of China (Grant \#31302167 and \#31372543).

\section{REFERENCES}

Brandsch M, Knütter I and Leibach FH (2004). The intestinal H+/peptide symporter PEPT1: structure-affinity relationships. Euro. J. Pharm. Sci. 21: 53-60.

Dalmasso G, Nguyen HTT, Yan Y, Charrier-Hisamuddin L, et al. (2008). Butyrate transcriptionally enhances peptide transporter PepT1 expression and activity. PLoS One 3: e2476.

Fotiadis D, Kanai Y and Palacín M (2013). The SLC3 and SLC7 families of amino acid transporters. Mol. Aspects Med. 34: $139-158$.

Li H, Gilbert ER, Zhang Y, Crasta O, et al. (2008). Expression profiling of the solute carrier gene family in chicken intestine from the late embryonic to early post-hatch stages. Anim. Genet. 39: 407-424.

Liu Z, Zhou Y, Liu S, Zhong H, et al. (2012). Characterization and dietary regulation of glutamate dehydrogenase in different ploidy fishes. Amino Acids 43: 2339-2348.

Maruska KP and Fernald RD (2011). Social regulation of gene expression in the hypothalamic-pituitary-gonadal axis. Physiology 26: 412-423.

Mylonas CC, Fostier A and Zanuy S (2010). Broodstock management and hormonal manipulations of fish reproduction. Gen. Comp. Endocrinol. 165: 516-534.

Shen H, Smith DE and Brosius FC 3rd (2001). Developmental expression of PEPT1 and PEPT2 in rat small intestine, colon, and kidney. Pediatr. Res. 49: 789-795.

Sherriff RM, Broom MF and Chadwick VS (1992). Isolation and purification of N-formylmethionine aminopeptidase from rat intestine. Biochim. Biophys. Acta 1119: 275-280. 
Sire MF and Vernier JM (1992). Intestinal absorption of protein in teleost fish. Comp. Biochem. Physiol., Part A Mol. Integr. Physiol. 103: 771-781.

Zohar Y and Mylonas CC (2001). Endocrine manipulations of spawning in cultured fish: from hormones to genes. Aquaculture 197: 99-136. 